Copyright C1997, American Institute of Aeronautics and Astronautics, Inc.

AIAA Meeting Papers on Disc, January 1997

A9715694, AIAA Paper 97-0665

\title{
Initial operation of a high-pressure detonation-driven shock tube facility
}

W. S. Stuessy

Texas Univ., Arlington

Hsuan-Cheng Liu

Texas Univ., Arlington

Frank K. Lu

Texas Univ., Arlington

Donald R. Wilson

Texas Univ., Arlington

AIAA, Aerospace Sciences Meeting \& Exhibit, 35th, Reno, NV, Jan. 6-9, 1997

The Hypersonic Shock Tunnel at the University of Texas at Arlington has been converted from a pressure-driven to a detonation-driven shock tube. This modification was required to provide a high pressure, high temperature gas environment. The existing shock tunnel is described along with the modifications needed for the conversion. The predicted performance is compared with the actual experimentally measured performance. (Author) 


\title{
Initial Operation of a High-Pressure Detonation-Driven Shock Tube Facility
}

\author{
W. S. Stuessy , H.-C. Liu ${ }^{\dagger}$, Frank K. L. ${ }^{\dagger}$, and D. R. Wilson ${ }^{\$}$ \\ Aerodynamics Research Center \\ The University of Texas at Arlington \\ Arlington, Texas 76019
}

\begin{abstract}
The Hypersonic Shock Tunnel at the University of Texas at Arlington (UTA) has been converted from a pressure-driven to a detonation-driven shock tube. This modification was required to provide a high pressure, high temperature gas environment. The existing shock tunnel is described along with the modifications needed for the conversion. The predicted performance is compared with the actual experimentally measured performance.
\end{abstract}

\section{INTRODUCTION}

The performance of a shock tube ${ }^{\prime}$ or a shock tunnel can be enhanced by several means. A common method is to use a light gas such as helium in the driver section. Another method is to increase the temperature of the driver gas by use of a heater. Both methods improve performance without an increase in pressure in the driver tube. Using helium results in a lighter molecular weight of gas in the driver and a higher speed of sound. This results in a lower driver to driven tube pressure ratio required to generate a given incident shock Mach number in the driven tube compared to air as the driver gas, or a much higher incident shock Mach number for the same driver pressure. The down side of using helium is the higher specific heat ratio which reduces the advantage some what. Heating the gas also increases the speed of sound and improves performance. Both of these methods have disadvantages. High pressure pumps and plumbing are

"Faculty Research Associate, Senior Member

${ }^{\dagger}$ Graduate Research Associate, Student Member

† Associate Professor of Aerospace Engineering, Associate Fellow

$\$$ Professor of Aerospace Engineering, Associate Fellow

Copyright (C) 1997 by the American Institute of Aeronautics and Astronautics, Inc. All rights reserved. required. Helium is expensive and large amounts must be used to pressurize the driver tube. Heating the driver is also expensive as the driver must be designed to withstand the high pressure at elevated temperatures. The heater adds to the cost and it must be designed to heat uniformly because hot spots in the driver can cause premature and dangerous driver tube failure. Reducing the diameter of the driven tube with respect to the driver also increases performance.

Another approach to improve the performance of the shock tube is to use a detonation wave driver, especially by using oxygen and hydrogen mixtures with helium dilution. The benefits of this is that the driver gas, after detonation, achieves a high pressure, a high temperature, a low molecular weight, a low specific heat ratio, and a high speed of sound. The initial pressure of the gaseous mixture is quite low eliminating the need of high pressure equipment to pressurize the driver. The high temperature generated is for very short periods of time so the driver structure is not exposed to high temperature. The design is thereby reduced to one of pressure containment Disadvantages are the dangers involved with the explosive mixture and the cost of an ignition source The danger of the explosive mixture can be reduced by helium dilution. Helium dilution does not substantially degrade performance and in certain situations actually allow more control of the temperature generated in the driver. The molecular weight of the driver gas is reduced by the helium. The addition of helium slightly increases the energy required to cause a detonation This enhances safety without significantly increasing the cost as only a small amount of helium is used as compared to a pure helium driver at high pressure.

The University of Texas at Arlington is supporting MSE, Inc. in a NASA sponsored program to determine the feasibility of using MHD accelerator technolog! with high pressure plasma's. The range of interest is between 0.01 and $100 \mathrm{~atm}$ and $2200^{\circ}$ and $4000^{\circ} \mathrm{K}$ at about Mach 2. Modifying the existing shock tunncl to a detonation driver shock tube creates a facility which can produce these conditions in the non-reflected mode of shock tube operation. 


\section{FACILITY DESIGN \& PERFORMANCE ANALYSIS}

The detonation driver can be operated in two modes. The first is to locate the ignition source at the most upstream end of the driver so that the detonation wave propagates downstream towards the diaphragm and reflects of the diaphragm as it ruptures. A wave diagram showing the downstream propagation of the detonation wave is shown in Figure 1. The gas behind the reflected detonation wave initiates the incident shock wave in the driven tube. The second is to locate the ignition source at the downstream end of the driver near the diaphragm. This uses the lower pressure gas behind the detonation wave to rupture the diaphragm and initiate the incident shock wave in the driven tube, so the performance is, consequently, lower. The detonation wave will travel upstream towards the closed end of the driver then reflect as shown in Figure 2. The pressure behind the reflected detonation wave must still be kept below the pressure limit of the driver. The first mode uses this maximum pressure to initiate the incident driven tube shock while the second does not.

The downstream mode was chosen for the detonation driver. Design and performance analysis calculations for the detonation driven shock tunnel were made using the TEPTM computer code ${ }^{2}$, a Windows $^{\text {TM }}$ version of the NASA CEC76 code ${ }^{3}$. This code performs real-gas calculations of a variety of gasdynamics processes, including Chapman-Jouguet (C-J) detonation waves, shock waves, and isentropic nozzle performance. A quasi-one-dimensional flow model is assumed, and real gas calculations based on both equilibrium and frozen flow models are available. All of the shock tube performance calculations are based on equilibrium flow.

The TEPTM code was first used to calculate detonation tube performance for stoichiometric mixtures of hydrogen and oxygen, for a range of initial pressures and different amounts of helium dilution. An existing, perfect gas code ${ }^{4}$ calculated the driven-tube pressure ratio $p_{2} / p_{1}$ and shock speed as a function of the shock tube pressure ratio $\mathrm{p}_{4} / \mathrm{p}_{1}$, gas properties, and shock tube area ratio. The TEPTM code was then used to calculate the temperature ratio across the incident shock wave using the perfect gas value of shock speed. The predicted performance of the detonation driver, with and area ratio of 13.65, is shown in Figure 3. The performance envelope is obtained for a stoichiometric oxygen and hydrogen mixture at initial driver pressure ranging from $1 \mathrm{~atm}$ to $8 \mathrm{~atm}$ and a driven tube filled with air at initial pressure from $0.063 \mathrm{~atm}$ to $10 \mathrm{~atm}$. The initial gas temperature is $300^{\circ} \mathrm{K}$. Also included are the performance maps of cold air and cold helium drivers, filling the driver with up to its maximum rating of $400 \mathrm{~atm}$. The downstream propagating detonation wave mode of operation was chosen as it produced in excess of $100 \mathrm{~atm}$ and $4000^{\circ} \mathrm{K}$ bchind the incident shock wave. The upstream propagating detonation wave would not produce these conditions

\section{FACII,ITY}

\section{EXISTING SHOCK TUNNEL}

The existing shock tunnel facility ${ }^{5}$ is shown schematically in Figure 4. The shock tube is composed, of a $15.24 \mathrm{~cm}(6 \mathrm{in})$ diameter, $3 \mathrm{~m}$ (10 ft) long driver tube, and a $15.24 \mathrm{~cm}$ ( $6 \mathrm{in})$ diameter, $8.23 \mathrm{~m}(27 \mathrm{ft})$ long driven tube. The two are separated by a doublediaphragm section. Both tube sections are rated for pressure of $41.3 \mathrm{MPa}(6000 \mathrm{psi})$. The diaphragms are normally constructed from 10 or 12 gauge (3.42 or 2.66 mm) hot-rolled 1008 steel plates, scored to various depths in a cross pattern. A $7.5^{\circ}$ half-angle conical nozzle with interchangeable throat inserts for Mach numbers of 5 to 16 is attached to the end of the driven tube. Presently the nozzle is driven by the gas behind the reflected shock wave in the driven tube and produces a exit Mach number of 8 . The exil diameter of the nozzle is $33.6 \mathrm{~cm}$ (13.25 in). A secondary diaphragm constructed from $0.0127 \mathrm{~mm}(0.005 \mathrm{in})$ thick aluminum sheet is located in the nozzle throat region, and is used to separate the driven-tube gas from that in the test section. The test section is a semi-free jet design, $53.6 \mathrm{~cm}(21.1 \mathrm{in})$ long and $44.0 \mathrm{~cm}$ (17.5 in) in diameter. Two $23 \mathrm{~cm}$ ( 9 in) diameter optical windows are located on opposite sides of the test section. The diffuser is $30.5 \mathrm{~cm}$ (12 in) in diameter, $213 \mathrm{~cm}$ (84 in) long and connects the test section to a $4.25 \mathrm{~m}^{3}\left(150 \mathrm{ft}^{3}\right)$ vacuum tank. The test section and diffuser contain model mounting and instrumentation ports.

The pneumatic system consists of a Haskell model 55696 two-stage gas-driven booster pump capable of charging the driver tube to $41.3 \mathrm{MPa}(6000 \mathrm{psi})$. The Haskell pump is normally connected to the facility air compressor system, consisting of a Clark CMB-6 5stage air compressor, twin-tower desiccant drier, and 14.5 MPa (2100 psi) storage bottles. Alternatively, the Haskell pump can be fed from a manifold of $15.2 \mathrm{MPa}$ (2200 psi) helium storage bottles. The vacuum system consists of a Sargent-Welch model 1376 (300 l/min) pump used to evacuate the driven tube, a SargentWelch model $1396(2800 \mathrm{l} / \mathrm{min})$ pump used to evacuate the test section/diffuser/vacuum tank, and a vacuum pressure measurement system consisting of two Baratron type 127A pressure transducers and the 
associated valve system to enable full range coverage from 1000 to 0.001 Torr.

\section{DETONATION DRIVER}

The driver from the existing Hypersonic Shock Tunnel at the University of Texas at Arlington is used as the detonation driver with some modifications. The existing driver tube is constructed of 4100 series steel and is $15.24 \mathrm{~cm}$ ( 6 in.) in diameter and $3 \mathrm{~m}(10 \mathrm{ft}$.) long. The tube has a pressure rating of $44 \mathrm{MPa}(6000$ psi). Ports for four surface mounted pressure transducers were installed to monitor the behavior of the detonation wave. Ports were also installed for an ignition plug at each end. The tube had two ports for injecting gases. These were used for injecting hydrogen, oxygen, air, and helium as well as for vacuuming out the initial air and venting the combustible mixture in case of an aborted run. This allowed operation in either the upstream or downstream propagating detonation wave mode. The conversion to a detonation driver retains the same pressure rating.

\section{DRIVEN TUBE}

The driven tube has been replaced with a new tube of stainless steel type 304 with $4.12 \mathrm{~cm}$ (1.62 in.) internal diameter and $9 \mathrm{~m}$ (30 ft.) in length. The tube is commercially available and has a pressure rating of 19 $\mathrm{MPa}$ (2800 psi). The existing shock tube has a driven tube of the same internal diameter as the driver tube,

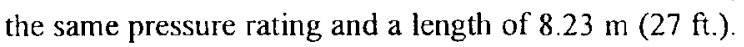
The existing driven tube is operated in a reflected mode and drives a nozzle capable of Mach numbers of between 5 and 16. The new driven tube is operated in a non-reflected mode and is therefor constructed to a lower pressure rating. Since the tube is operated in the non-reflected mode and the existing nozzle is designed for reflected operation, the nozzle is not used. The end of the driven tube is extended into an existing vacuum tank. The tank is not used for vacuum but is simply used to collect the exhaust before releasing it to atmosphere. This combination of driver and driven tube produces a driver-to-driven tube area of 13.65 which provides an improvement in performance.

\section{INJECTION SYSTEM}

The hydrogen, oxygen, and helium are injected through the sidewall of the driver. Two tubes are available for this purpose. The hydrogen and oxygen are injected through separate tubes for safety purposes. Helium and purge air are injected through the oxygen line. The initial air is evacuated through the hydrogen line and the combustible mixture is also vented through the hydrogen line. The two lincs also contain Matheson series 6103 flash arrestors for added safety.

\section{DATA ANALYSIS}

The electronic data from the pressure transducers is converted to pressure readings. The pressure levels are obtained directly and time of flight measurements are made by determining the time between passage of the detonation wave or shock wave between successive transducers. Since the transducers are a known distance apart, the shock or detonation velocity can be determined from measured time intcrval between adjacent pressure transducers. This provides an indication of the properties of the detonation wave, primarily that the wave has indeed transitioned to Chapman-Jouguet velocity.

\section{INSTRUMENTATION}

The detonation driver instrumentation includes four PCB model 111 A22 dynamic pressure transducers and a Baratron Pressure Transducer from MKS, model number 127A. The Baratron pressure transducer has a maximum pressure range of $1.33 \mathrm{MPa}$ (10000 Torr). This transducer is used to set the mixture ratio during filling of the detonation driver using partial pressures. The PCB transducers are mounted in the driver tube with the sensing diaphragm flush with the inside wall. Each one has a full scale range of $68.9 \mathrm{MPa}(10000$ psi), rise time of 2 microseconds, and a time constant of 1000 seconds. The Baratron is used to provide an initial pressure reading as the PCB's are dynamic transducers.

The driven tube instrumentation includes four $\mathrm{PCB}$ transducers. Two of these are PCB model $111 \mathrm{~A} 23$ which have a full scale pressure range of $34.4 \mathrm{MPa}$ (5000 psia), rise time of 2 microseconds, and a time constant of 500 seconds. These transducers are used primarily for shock speed measurements as they are upstream of the test area and separated by a precisely measured distance. The other two PCB transducers can be model 111A23 or 111A24 depending upon the conditions in the driven tube. The model 111A24 transducers have a full scale range of $6.89 \mathrm{MPa}(1000$ psia), a response time of 2 microseconds, and a time constant of 100 seconds. The initial pressure in the driven tube is also measured by a Baratron Pressure Transducer from MKS, model number 127A. This transducer has a maximum pressure range of $133 \mathrm{kPa}$ (1000 Torr). This transducer provides a very accurate measure of the initial driven tube pressure and provides an initial pressure for the dynamic PCB pressure transducers.

The pressure transducers are connected to a DSP Technology data acquisition system which has 48 channels capable of $100 \mathrm{kHz}$ sampling rate, 12 bits of 
accuracy, with each channel having its own amplifier and analog to digital converter to allow for simultaneous sampling of all channels. The system has 512 kilosamples of memory available for distribution between the channels being utilized. Eight channels are also available with the capability of $1 \mathrm{MHz}$ sampling rate, 12 bits of accuracy, and also with separate analog to digital converters for each channel. Two Megasamples of memory are available for these eight channels. The data acquisition system is controlled by a PC which retrieves the data through a IEEE-488 interface. The data is then stored on the harddrive for later analysis.

\section{EXPERIMENTAL TEST PROGRAM}

\section{FACILITY CHECKOUT}

The initial operation of the modified shock tube consisted of a series of test runs at increasing pressure levels to verify the design and refine operating procedures. The first test run was with a stoichiometric mixture of hydrogen and oxygen in the driver at $1 \mathrm{~atm}$. The driven tube contained atmospheric air. This pressure level was chosen to check the behavior of the detonation wave in the driver. The pressure levels measured and the wave speed calculated from the time between sensors were carefully analyzed and compared to data obtained at the same pressure in a Pulse Detonation Engine (PDE) Facility at the University. The PDE facility is much smaller but provides a wealth of experience and data. The initial operation had the ignition plug near the primary diaphragm to operate in an upstream operating mode. The initial driver pressure was then increased incrementally up to $6 \mathrm{~atm}$. The driver section was separated from the driven tube by a $0.381 \mathrm{~mm}(0.015 \mathrm{in})$ thick mylar diaphragm for all the tests. Thin mylar could be used since the initial pressure is low and the post detonation pressure is about 20 times the initial level.

\section{SHOCK TUBE PERFORMANCE}

The performance of the modified shock tube was next mapped out by varying the driven tube pressure along with the driver pressure. The performance was considerably less than predicted. The data from all the test runs were consistent so a gasdynamic phenomena was suspected of causing the lack of performance.

The ignitor plug was next moved to the upstream end of the driver. Several test runs were obtained at similar initial conditions as previous runs with the ignitor near the diaphragm. Comparing the data illustrated that the downstream ignition location provided better performance, contrary to the predicted performance.
The ignitor plug was returned to its original location. Next the driver initial pressure was increased to 7 and 8 atm to improve performance. The driver tube of our facility has a pressure limit of $400 \mathrm{~atm}$ and the reflected detonation wave in a stoichiometric mixture of hydrogen and oxygen at $8 \mathrm{~atm}$ produces $399 \mathrm{~atm}$. Driven tube shock Mach numbers increased with the higher pressure but not to the desired range.

Next helium was introduced to the combustible mixture. Varying amounts of helium $w$ as added to try to improve performance. The best performance was found using the ratio of 1 to 1.5 moles helium to 1 mole of oxygen to 2 moles of hydrogen. The performance again increased but it was still substantially below predicted levels

The experimental temperature versus pressure map is shown in Figure 5 . The measured shock Mach numbers are plotted in Figure 6 as a function of the initial pressure ratio of pre-detonation driver pressure divided by the initial driven tube pressure. The higher shock Mach numbers are generated with the lowest initial pressure in the driven tube as the theory predicts.

The detonation tube operated close to expectations for all cases. Figures 7 and 8 show the detonation wave pattern for the upstream propagation mode for two different initial driver pressures of 6 and 8 atm respectively. The first sensor detects some precompression ahead of the detonation wave. This is an indication that the detonation wave has not reached full C-J velocity. This precompression phenomena was also observed in the experiments reported by Helman ${ }^{6}$ The next sensor passed by the detonation wave does not detect any precompression and the time of flight calculations indicate the detonation wave has reached C.J velocity.

The downstream propagation mode is shown in Figure 9 for an initial pressure of $6 \mathrm{~atm}$. The time of flight measurement indicate $C-J$ velocity and the pressure traces show no sign of precompression. The peak detonation pressures are comparable to those obtained in the upstream propagating mode of operation but the overall shock tube performance measured by incident shock speed is less.

The driven tube pressure traces in Figures 10 and 11 are quite different. The lower initial driven tube pressure case in Figure 10 contain the initial pressure rise from the incident shock, followed by about $250 \mu \mathrm{s}$ of steady pressure, and then a gradual rise in pressure. The gradual rise is caused by the tail of the expansion wave generated by the rupture of the diaphragm moving down the driven tube. The pressure then stabilizes for a short time before another shock is observed which is caused by the reflected detonation wave reaching the diaphragm location and creating 
another shock in the driven tube. The higher initial driven tube pressure case in Figure 11 contains the initial pressure rise from the incident shock, followed immediatcly by a very gradual pressure decrease until a sccond shock wave comes down the driven tube. The gradual pressure decrease is a result of the expansion process following the detonation wave in the driver propagating into the driven tube behind the incident shock and weakening it and the pressure generated behind it.

\section{PERFORMANCE COMPARISON}

The measured performance of the detonationdriven shock tube fell considerably below the anticipated performance for both upstream and downstream propagating modes. An analysis of the test data, coupled with experience gained from basic detonation wave experiments conducted as part of a Pulse Detonation Wave Engine (PDE) development program, suggest the following reasons for the discrepancy.

Figure 1 illustrates the wave process for the "downstream" mode of operation, resulting from ignition of the detonable mixture at the upstream end of the detonation tube. The incident detonation wave is followed by a strong expansion wave that is generated to satisfy the zero velocity boundary condition at the closed end of the tube. This expansion wave causes an immediate drop in pressure from the Chapman-Jouguet level generated by the incident detonation wave, and the interacting of this expansion wave with the reflected detonation wave appears to drastically lower the pressure level behind the reflected detonation wave. It is this pressure that initiates and sustains the incident shock wave propagation in the driver tube, and consequently very rapid attenuation of the incident shock wave is suspected.

A different cause is suspected for the reduced performance with the "upstream" propagation mode of opcration. Figure 8 shows typical pressure traces in the detonation tube for this mode. The important point to notice is that the initial wave formation is a weak detonation wave $\left(\mathrm{M}_{\mathrm{j}} \sim 1.7\right)$ that transitions to a fully-developed $\mathrm{CJ}$ detonation wave $\left(\mathrm{M}_{\mathrm{D}} \sim 5.3\right)$ about $50 \mathrm{~cm}(20 \mathrm{inch})$ from the ignition source. Figure 12 shows an endwall pressure trace from a similar test conducted as part of our PDE development program. This data was from a $7.62 \mathrm{~cm}$ ( 3 in) diameter chamber continuing a stoichiometric mixture of hydrogen and oxygen, initially at a pressure level of I atm. Transition to a fully developed CJ detonation wave for this case was triggered by a Shchelkin spiral ${ }^{7}$ inserted into the detonation chamber, but similar results have been observed for cases at higher initial pressure or with different fuels which naturally transitioned to $C J$ detonation wave propagation speeds without turbulence enhancement. The end-wall pressure is seen to gradually increase to a level of about 70 psia, and then abruptly increase to a level of about 110 psia, corresponding to the transition in wave propagation from a weak to a $\mathrm{CJ}$ detonation wave. This pressure level is in close agreement with the predicted end-wall pressure for the ZND model, but the fact, that it does not form immediately upon ignition probably interferes with the formation of a strong incident shock wave in the driven tube.

\section{CONCLUSIONS \& RECOMMENDATIONS}

The reduced performance of the detonationdrivers shock tube prevented attainment of the shock Mach numbers required to meet the original goals of the program. We are currently modifying the facility to incorporate a helium driver for ignition of the $\mathrm{H}_{2} / \mathrm{O}_{2}$ mixture. This is similar to the concept incorporated by Bakos, et.al ${ }^{8}$ of GASL to expand the performance of the HYPULSE facility, and we are indebted to Drs. Robert Bakos and John Erdos of GASL for many helpful discussions concerning this mode of operation. The concept is illustrated in Figure 13 (from Ref. 8) in which a high pressure helium driver is placed upstream of the detonation tube and used to drive a shock wave into the detonation tube. This shock wave should quickly transition to a CJ detonation wave, but the rarefaction wave associated with the closed-end mode of operation of the detonation tube should be drastically reduced, thus resulting in a much higher pressure level behind the detonation wave. In fact, in the "perfectly-driven" mode of operating, the full CJ pressure level can ideally be mainlained behind the detonation wave in Figure 14 (from Ref. 8).

5

American Institute of Acronautics and Astronautics 
This modification should result in significant performance improvements for the facility.

\section{ACKNOWLEDGMENTS}

The authors would like to acknowledge the support of MSE, Inc. through Contract No. 96-C323-F and recognize the contributions of $\mathrm{Dr}$. Y. M. Lee and Mr. Gloyd Simmons of MSE to the effort described in this paper. Also, valuable suggestions from Drs. John Erdos and Robert Bakos of GASL and Dr. Dave Bogdanoff of Eloret-Thermal Sciences Institute are gratefully acknowledged.

\section{REFERENCES}

1. Anderson, J. D., Jr., Modern Compressible Flow, 2nd-Ed., McGraw-Hill Publishing Co., New York, 1982

2. "Thermal Equilibrium Program for Window User's Manual," Software and Engineering Associates, Inc., 1992.

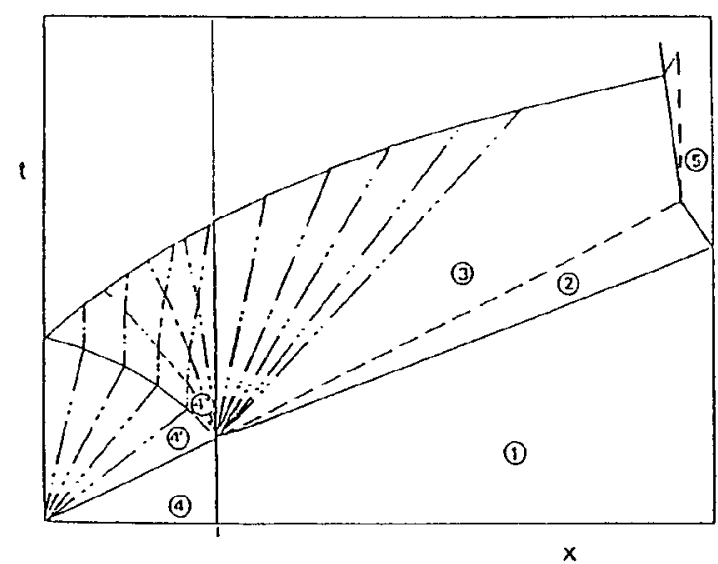

Figure 1 Downstream propagating

detonation wave diagram
3. Gordon, S. and McBride, B. J., "Computer Program for Calculation of Complex Chemical Equilibrium Composition, Rocket Performance, Incident and Reflected Shocks, and Chapman-Jouguet Detonations," NASA SP-273, 1976

4. Angelone, J.P., "Shock Tube-High Temperature Gasdynamics Studics," M.S. Thesis, The University of Texas at Arlington, 1978.

5. Stuessy, W.S., Murtuguddee, R. G., Lu, F. K., Wilson, D. R., "Development of the UTA Hypersonic Shock Tunnel," AIAA Paper 90-0090, 1990.

6. Helman, D., Shreeve, R. P, and Eidelman, S., "Detonation Pulse Engine," AIAA Paper 86-1683, 1986.

7. Shchelkin, K. I., Soviet Journal of Technical Physics, Vol 10, 1940, pp. 823-827.

8. Bakos, R. J., Castrogiovanni, A., Calleja, J. F., Nucci, L., and Erdos, J. I., "Expansion of the Scramjet Ground Test Envelope of the HYPULSE Facility," AIAA Paper 96-4506, 1996.

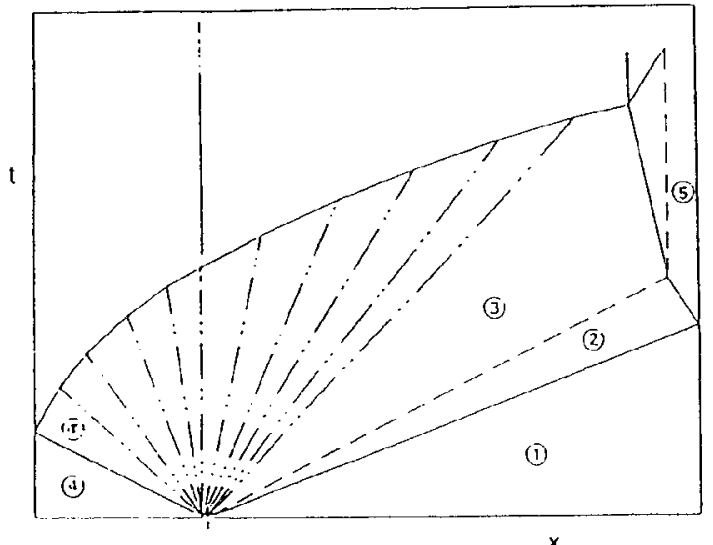

Figure 2 Upstream propagating

\section{detonation wave diagram}

6 
Copyright (C)1997, American Institute of Aeronautics and Astronautics, Inc.

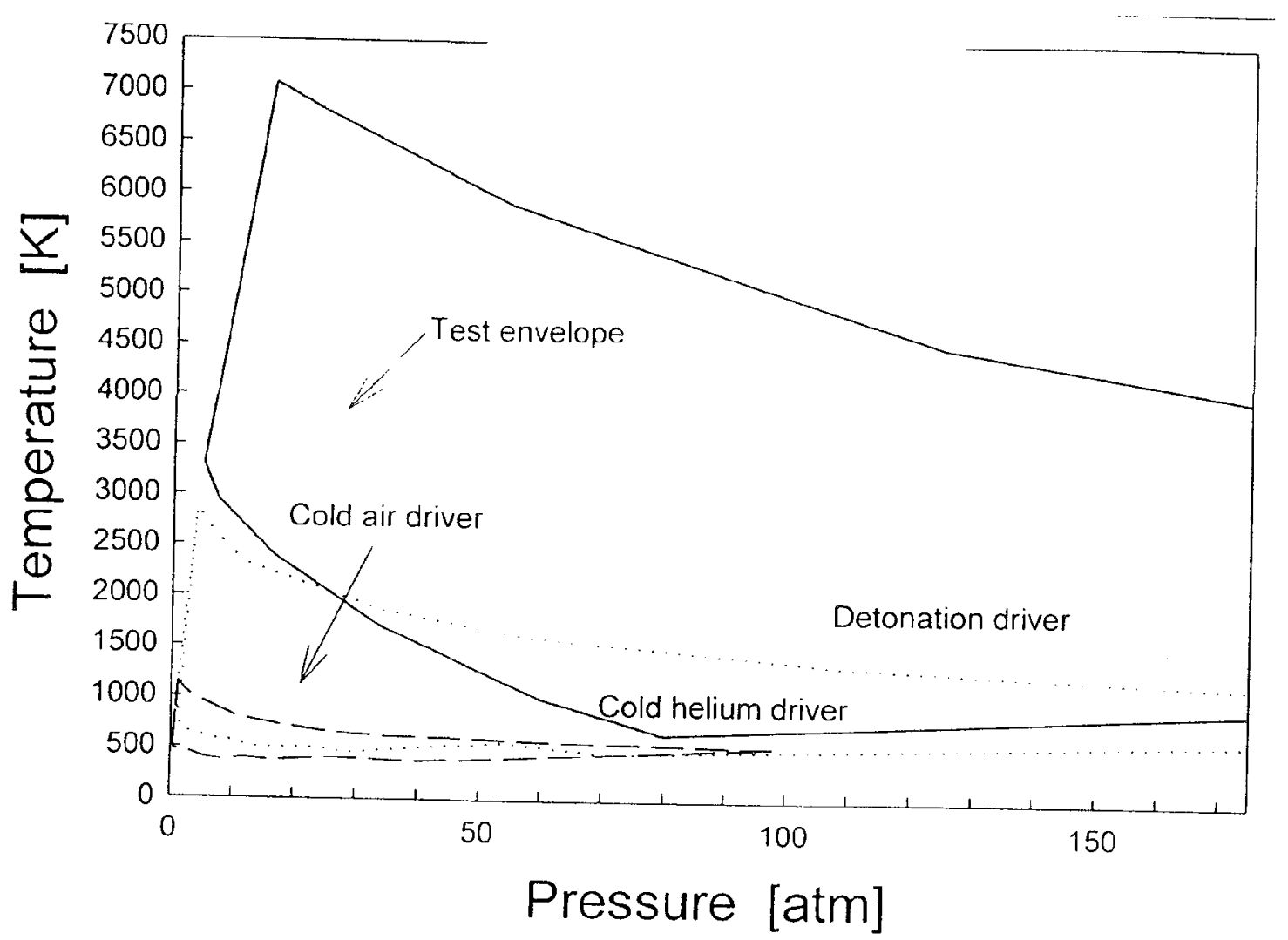

Figure 3 Predicted performance map

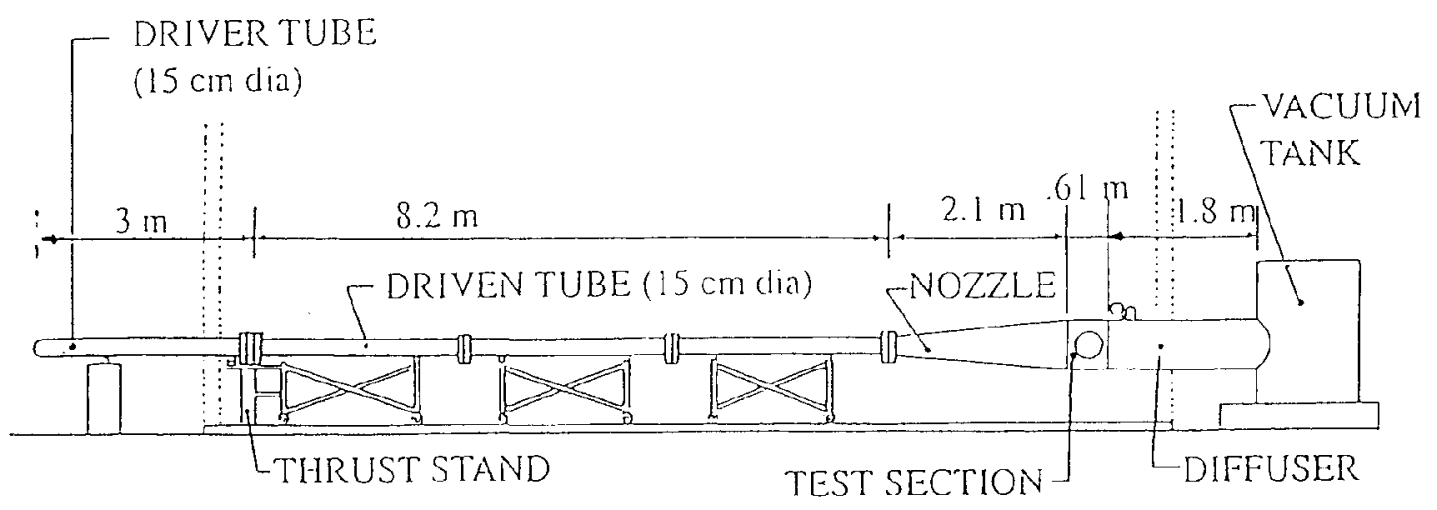

Figure 4 HST existing elevation view 
Copyright (c)1997, American Institute of Aeronautics and Astronautics, Inc.
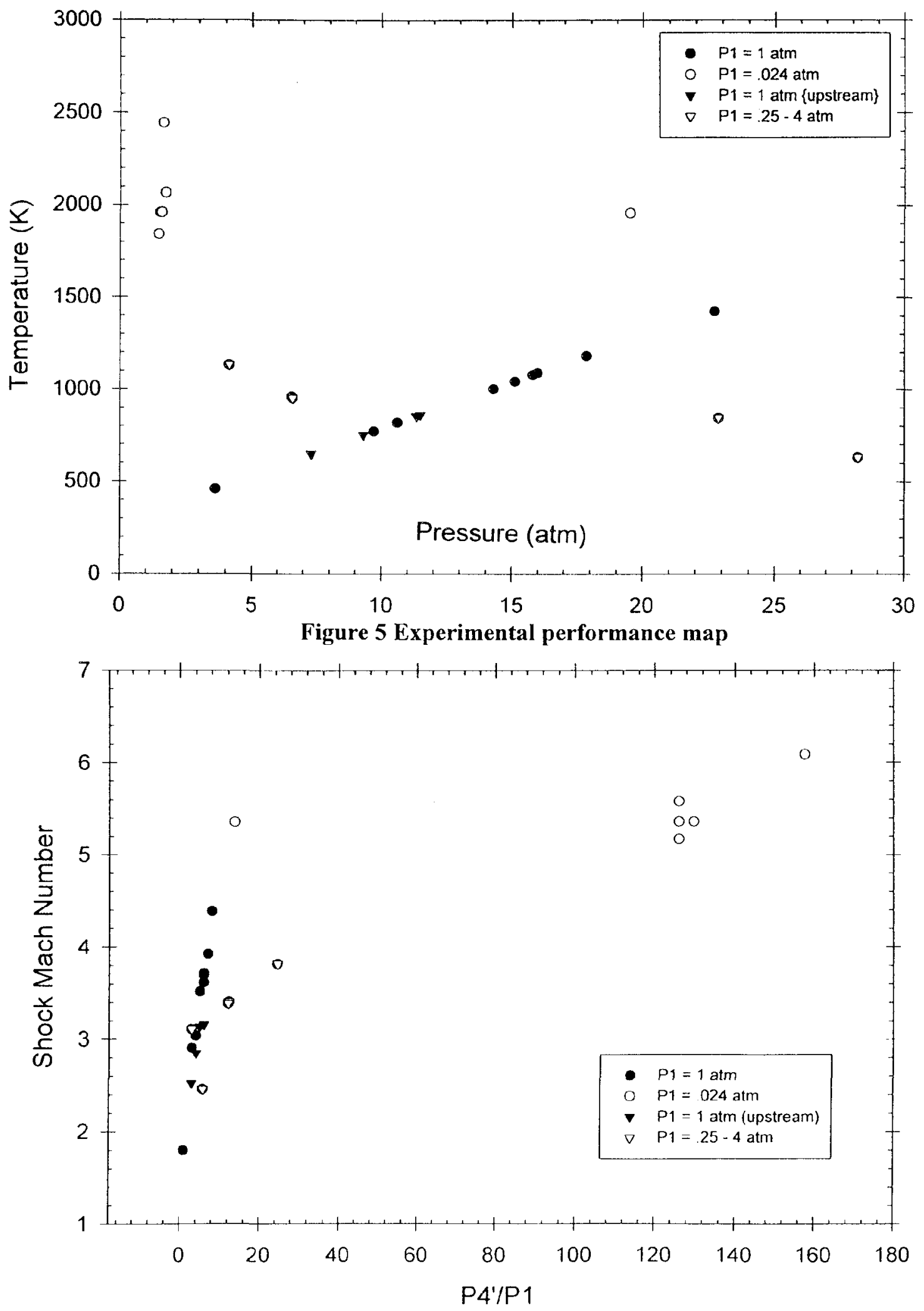

Figure 6 Experimental shock mach number map 
Copyright (C1997, American Institute of Aeronautics and Astronautics, Inc.

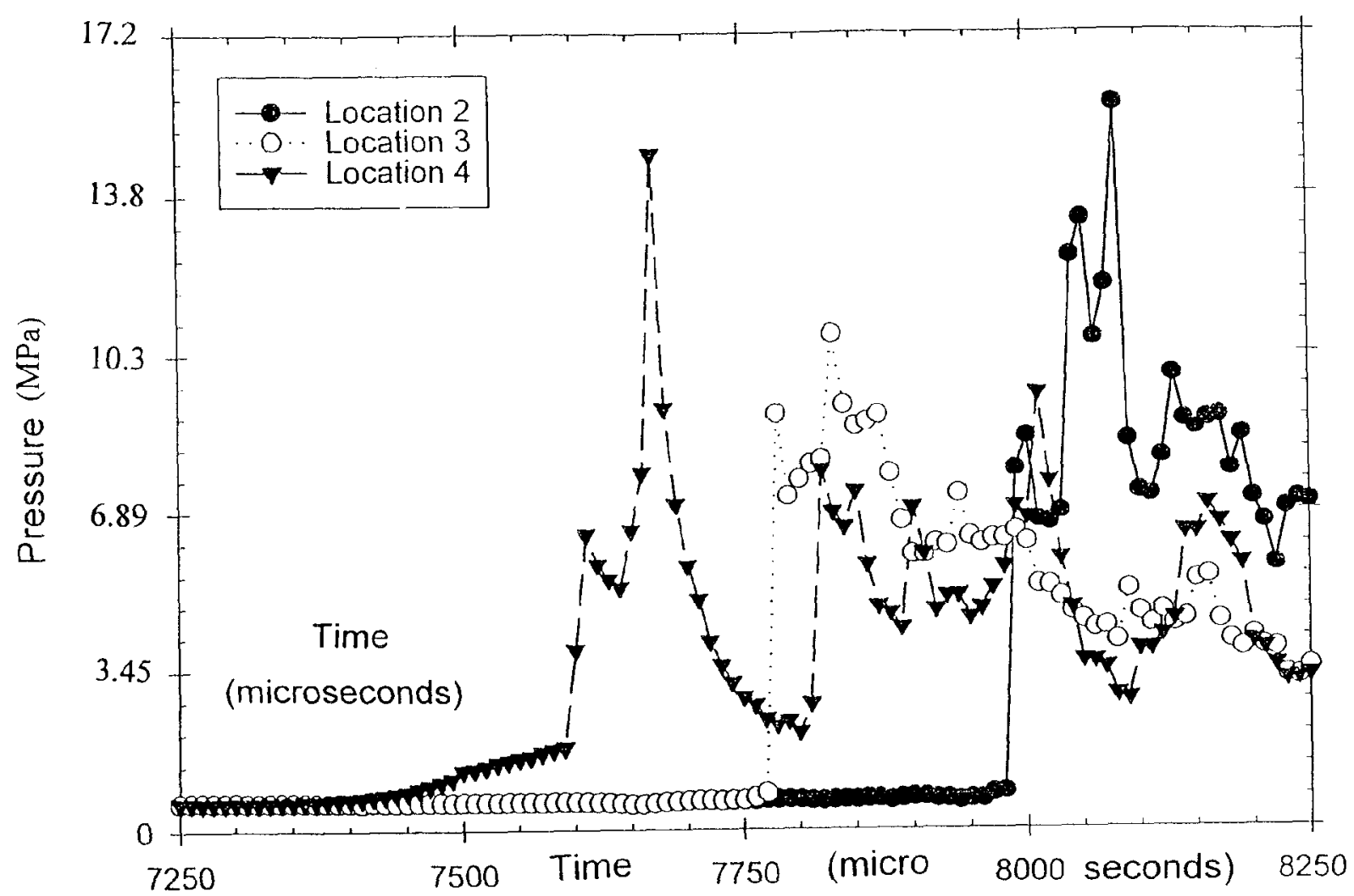

Figure 7 Pressure trace upstream propagating detonation, 6 atm initial pressure

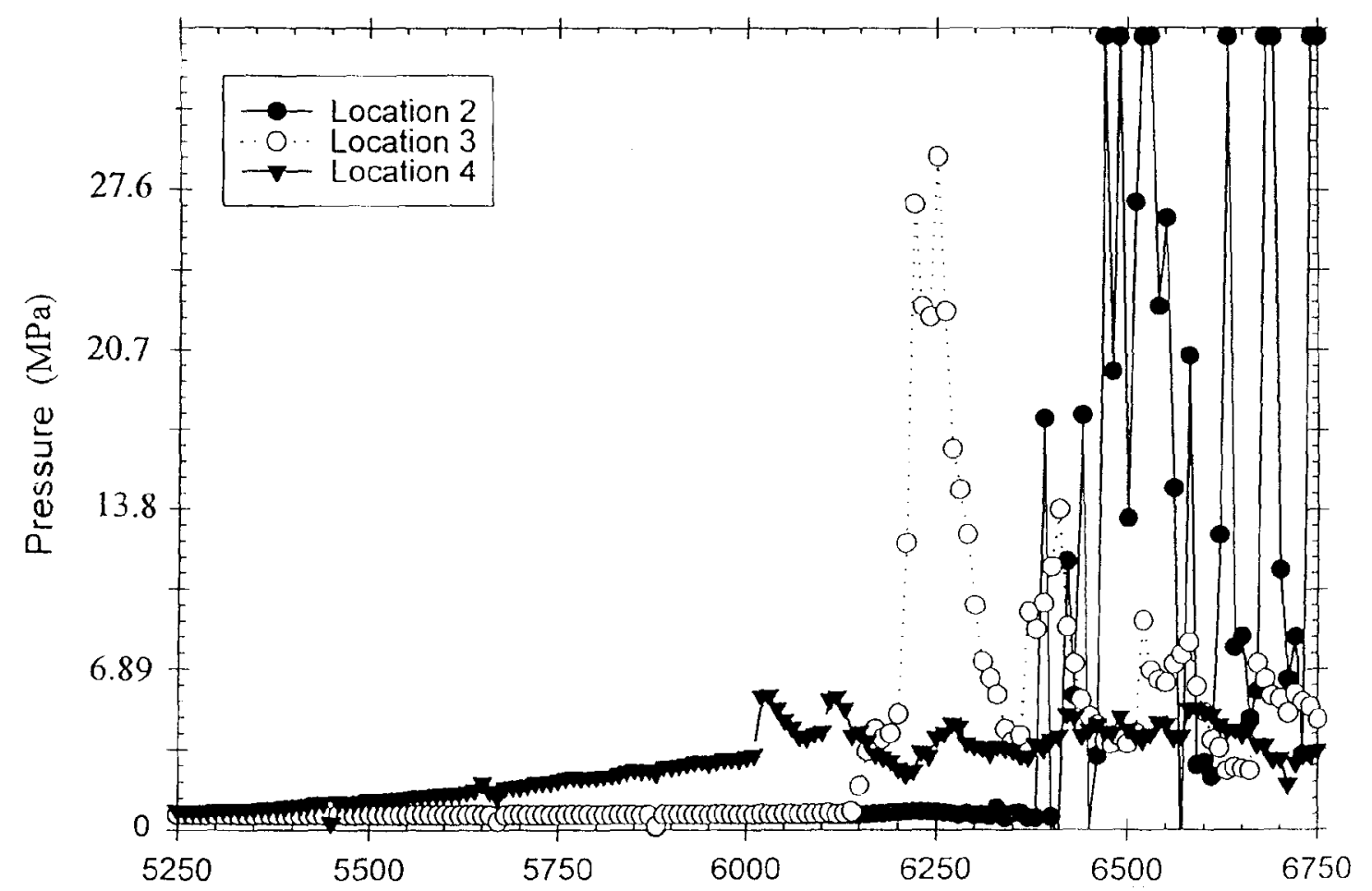

Figure 8 Pressure trace upstream propagating detonation, 8 atm initial pressure 


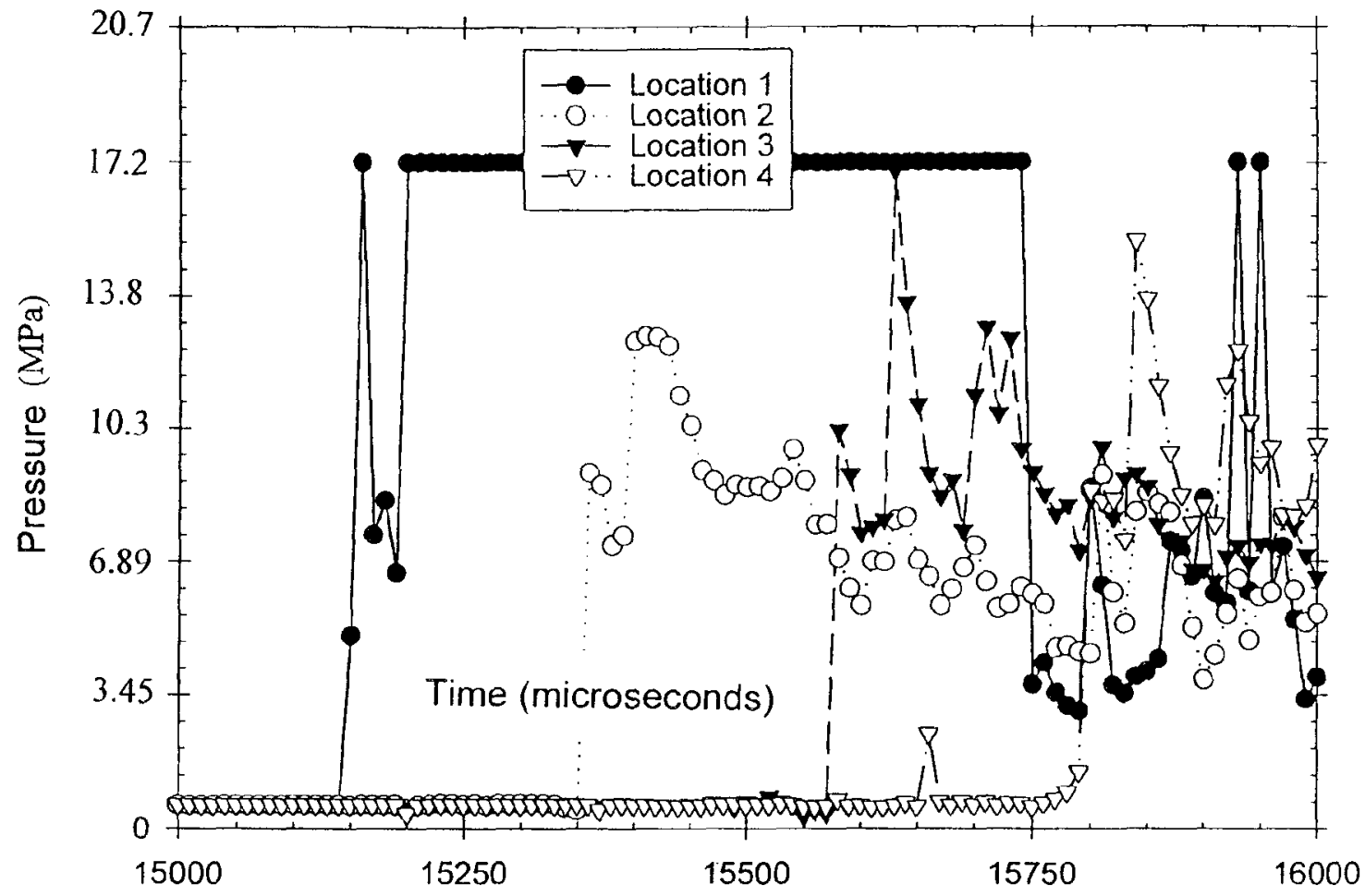

Figure 9 Pressure trace downsteam propagating detonation, 6 atm initial pressure

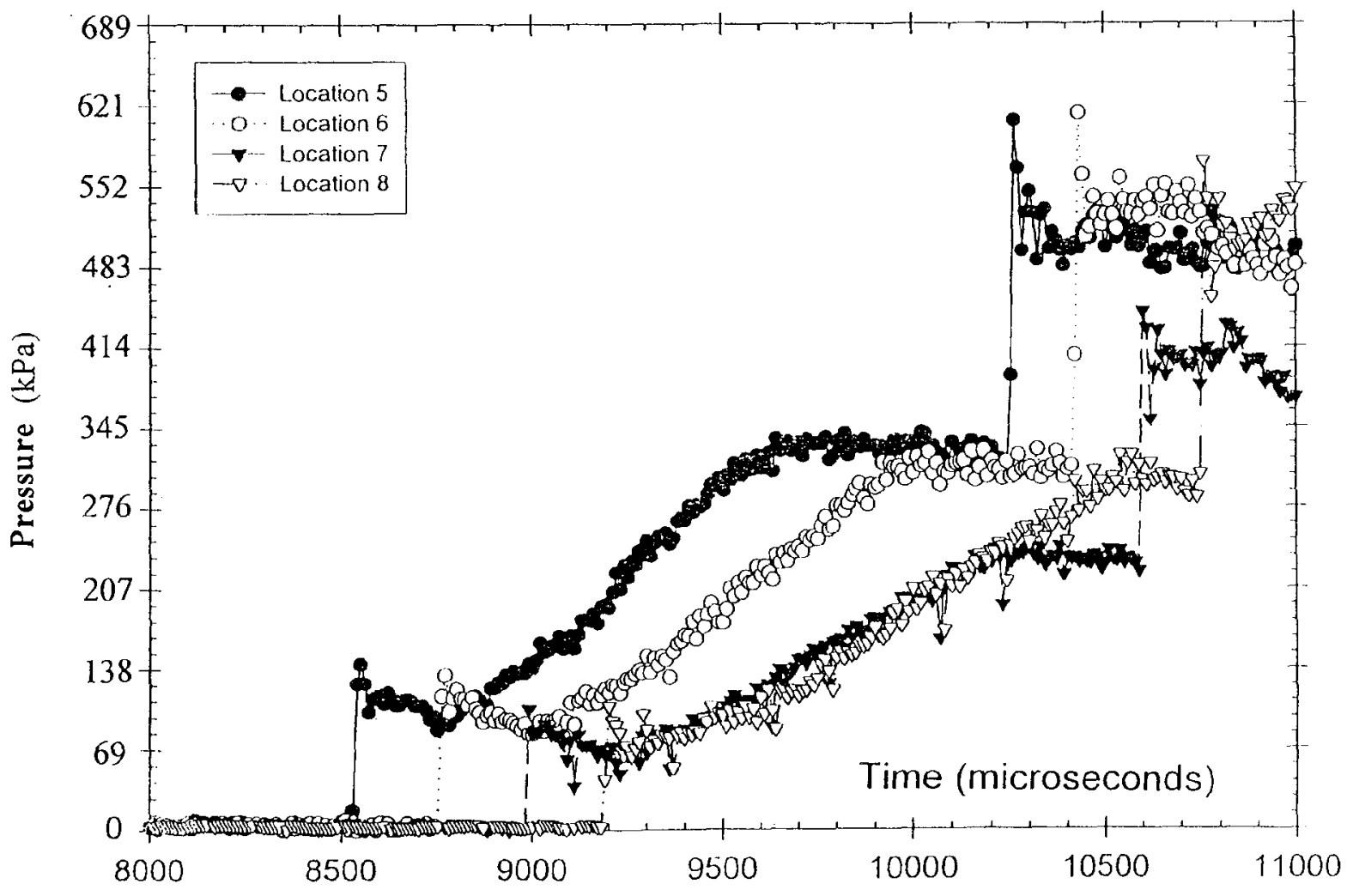

Figure 10 Driven pressure low initial pressure 
Copyright (C1997, American Institute of Aeronautics and Astronautics, Inc.

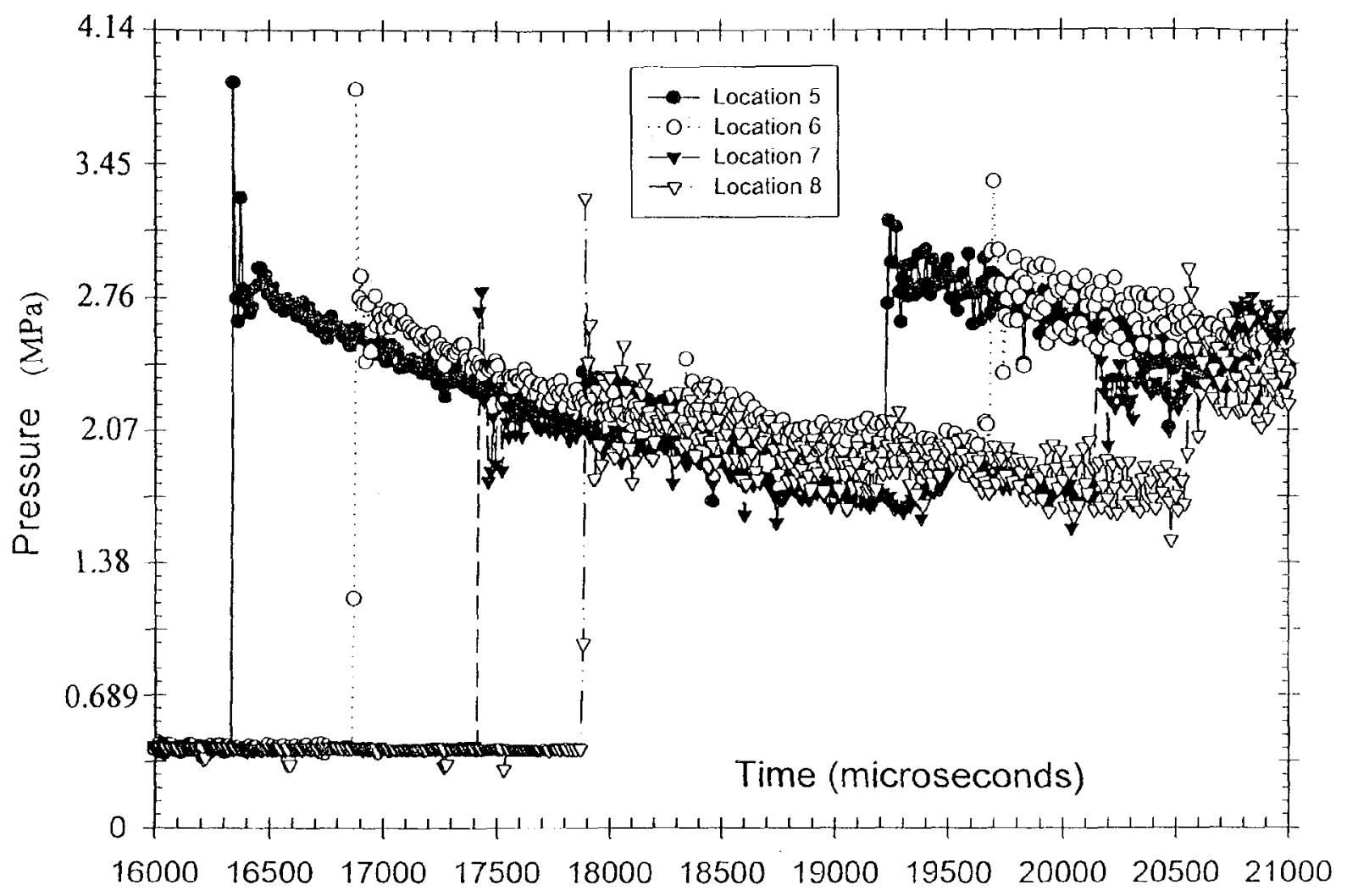

Figure 11 Driven pressure high initial pressure

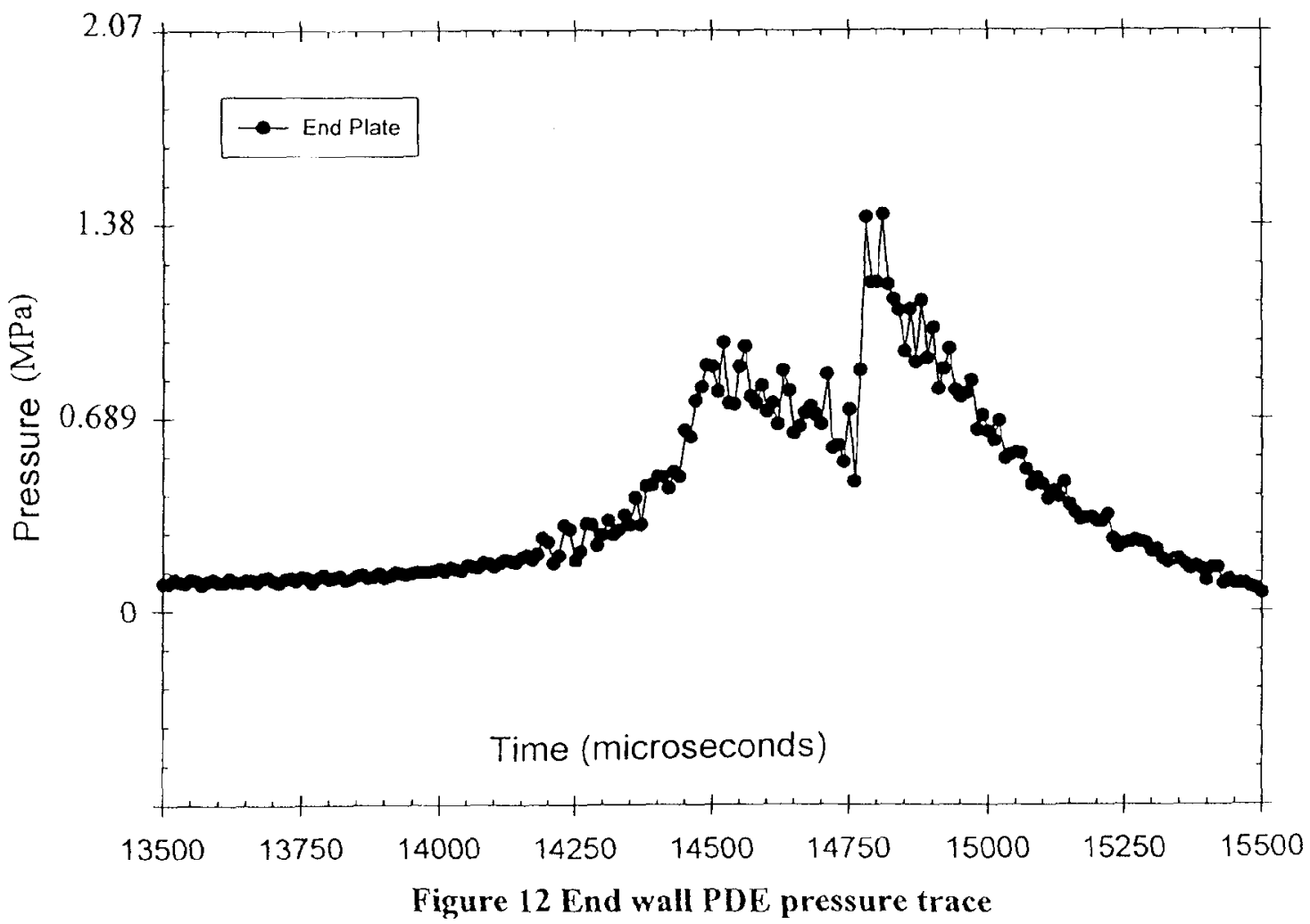

American Institute of Aeronautics and Astronautics 


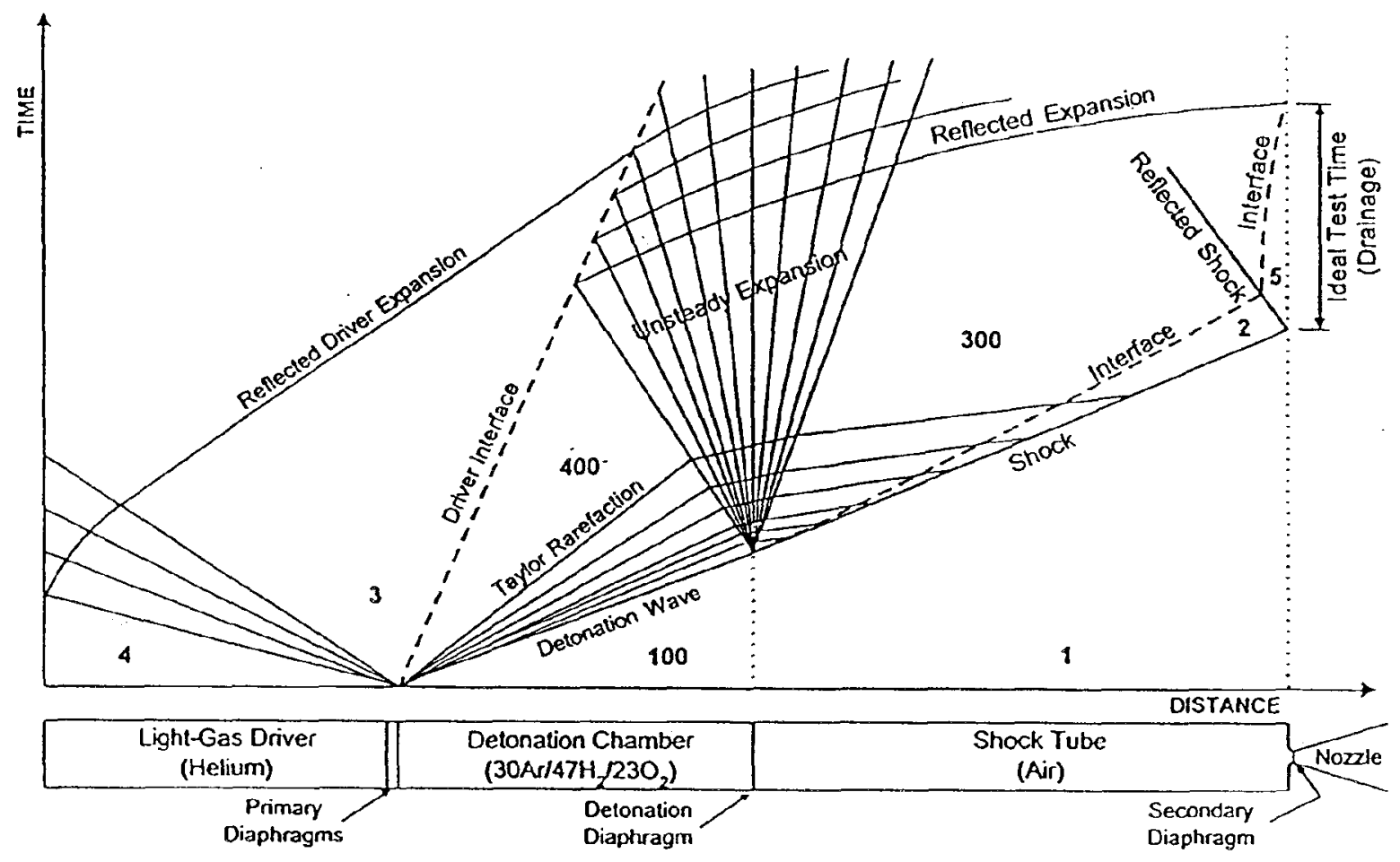

Figure 13 Helium driven detonation driver wave diagram (from Ref. 8)

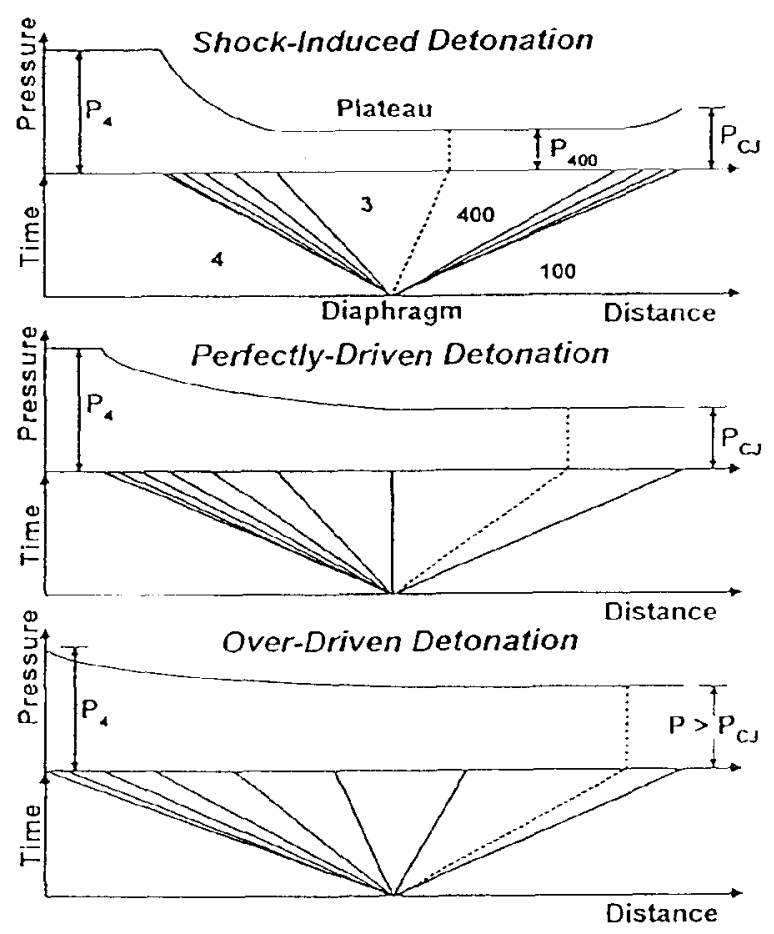

Figure 14 Helium driven detonation driver pressure diagrams ( from Ref. 8) 